\title{
Painful fingertip swelling of the middle finger
}

\author{
Fabio Becce • Biljana Jovanovic • Louis Guillou • \\ Nicolas Theumann
}

Received: 22 March 2011 /Revised: 15 May 2011 / Accepted: 20 May 2011 /Published online: 18 June 2011

(C) ISS 2011

Keywords Finger osteoid osteoma radiographs · curettage

\section{Diagnosis}

Osteoid osteoma of the distal phalanx of the middle finger.

\section{Discussion}

Osteoid osteoma (OO) is a benign bone-forming tumour that accounts for about $12 \%$ of benign and $3 \%$ of all bone neoplasms [1]. It mainly affects patients during childhood or early adulthood, with a male preponderance. Despite its predilection for the long bones of the lower limbs, this tumour can occur in any bone of the skeleton and may be found in the hand and wrist in up to $10 \%$ of cases [2]. However, OOs of the distal phalanx are relatively uncom-

The case presentation can be found at doi:10.1007/s00256-011-1218-z

F. Becce $\cdot N$. Theumann $(\bowtie)$

Department of Diagnostic and Interventional Radiology,

Centre Hospitalier Universitaire Vaudois, University of Lausanne,

Rue du Bugnon 46,

1011 Lausanne, Switzerland

e-mail: nicolas.theumann@chuv.ch

B. Jovanovic

Plastic and Hand Surgery Centre,

1003 Lausanne, Switzerland

L. Guillou

University Institute of Pathology,

Centre Hospitalier Universitaire Vaudois, University of Lausanne,

1011 Lausanne, Switzerland mon, with only a few case series reported in the English literature so far [3-5].

The diagnosis of distal phalangeal $\mathrm{OO}$ is challenging for several reasons. First, the typical clinical features may not be present $[2,3,5]$. Pain may not be worse at night, or be relieved by aspirin. Moreover, soft-tissue swelling and erythema, as well as nail deformities, may mislead the physician. Second, its imaging characteristics (i.e. a small radiolucent lesion with central calcification, peripheral sclerosis and periosteal reaction [1]) may also be atypical [3-6]. Because of the presence of thickened Sharpey's fibres, a periosteal reaction is rarely found in the distal phalanx [6]. Besides, endosteal sclerosis may be counterbalanced by local osteopaenia. Furthermore, other bony lesions of the fingertips, such as chronic osteomyelitis, epidermoid cyst, enchondroma or glomus tumour, may mimic an $\mathrm{OO}[3,5,6]$. Zampa et al. recently reported the added value of dynamic contrast-enhanced MR imaging for $\mathrm{OO}$ in atypical locations, in terms of nidus conspicuity and diagnostic confidence [7]. Third, the histological features may even be unusual [5]. All those reasons could explain the delay, sometimes of several months to years, in the diagnosis and the treatment of phalangeal OOs.

The main clinical differential diagnosis of painful fingertip swelling includes whitlow or paronychia, osteomyelitis, glomus tumour, subungual exostosis, arthritis and OO $[3,5,6]$. In our case, conventional radiographs (see Fig. 1 in the Question), ultrasound and previous CT and MR imaging studies (all not shown) barely displayed a focal lesion at the base of the distal phalanx. Follow-up MRI, performed at our institution several months after the onset of symptoms and after failure of initial surgery, demonstrated persisting diffuse inflammatory changes of the distal and middle phalanges as well as in the 
surrounding soft tissues (see Fig. 2a, b). On fourdimensional MR angiographic images, the lesion was brightly enhanced in the arterial phase (see Fig. 2c). On dynamic MR angiography, OO typically has a peak enhancement in the arterial phase with an early partial washout, compared with the slower progressive enhancement of the adjacent bone marrow (see Fig. 2c, d) [7]. Except for the glomus tumour, other bony lesions may not exhibit this pattern of enhancement. The calcification of the nidus was better noted on the following CT (see Fig. 3) and the diagnosis of $\mathrm{OO}$ was later confirmed at histopathology (see Fig. 4).

Percutaneous CT-guided ablations with laser [8] or radiofrequency [9] have now widely replaced surgery as the treatment of choice for OO. However, surgical excision still plays a major role for the lesions in the hands and feet owing to the close relationship of the small bones with the neurovascular structures [9, 10]. Indeed, a few complications have been reported after radiofrequency ablation of phalangeal OOs [10]. The patient we present benefited from surgical curettage of his lesion at the base of the distal phalanx. Percutaneous treatment could not be performed due to the intra-articular location of the OO, with the ungual matrix and the insertion of the flexor digitorum profundus tendon on respectively the dorsal and volar aspects of the distal interphalangeal joint. The function of the distal interphalangeal joint could be preserved and the symptoms completely disappeared a few days after surgery, with no sign of recurrence more than a year after treatment.

In conclusion, distal phalangeal $\mathrm{OO}$ is uncommon, but should be included in the differential diagnosis of painful swelling of the fingertip. Dynamic contrast-enhanced MR imaging is useful to detect and to differentiate this tumour from OO-mimicking lesions. Surgery is still the treatment of choice for phalangeal OOs because of their close relationship with the neurovascular structures.

\section{References}

1. Becce F, Theumann N, Rochette A, et al. Osteoid osteoma and osteoid osteoma-mimicking lesions: biopsy findings, distinctive MDCT features and treatment by radiofrequency ablation. Eur Radiol. 2010;20:2439-46.

2. Ambrosia JM, Wold LE, Amadio PC. Osteoid osteoma of the hand and wrist. J Hand Surg Am. 1987;12:794-800.

3. Foucher G, Lemarechal P, Citron N, Merle M. Osteoid osteoma of the distal phalanx: a report of four cases and review of the literature. J Hand Surg Br. 1987;12:382-6.

4. Meng QF, Watt I. Phalangeal osteoid osteoma. Br J Radiol. 1989;62:321-5.

5. Burger IM, McCarthy EF. Phalangeal osteoid osteomas in the hand: a diagnostic problem. Clin Orthop Relat Res. 2004;427:198-203.

6. Jones SN, Stoker DJ. Radiology at your fingertips; lesions of the terminal phalanx. Clin Radiol. 1988;39:478-85.

7. Zampa V, Bargellini I, Ortori S, Faggioni L, Cioni R, Bartolozzi C. Osteoid osteoma in atypical locations: the added value of dynamic gadolinium-enhanced MR imaging. Eur J Radiol. 2009;71:527-35.

8. Zouari L, Bousson V, Hamzé B, Roulot E, Roqueplan F, Laredo JD. CT-guided percutaneous laser photocoagulation of osteoid osteomas of the hands and feet. Eur Radiol. 2008;18:2635-41.

9. Soong M, Jupiter J, Rosenthal D. Radiofrequency ablation of osteoid osteoma in the upper extremity. J Hand Surg Am. 2006;31:279-83.

10. Harrod CC, Boykin RE, Jupiter JB. Pain and swelling after radiofrequency treatment of proximal phalanx osteoid osteoma: case report. J Hand Surg Am. 2010;35:990-4. 